\section{Large-scale Field Trials of Imidacloprid for Control of the Spined Citrus Bug}

\author{
J. Mo ${ }^{1}$ and K. Philpot \\ Yanco Agricultural Institute, PMB Yanco, NSW 2703, Australia
}

Additional index words. endosulfan withdrawal, alternative insecticide, beneficials, lemon, Biprorulus bibax

\begin{abstract}
Four large-scale field trials were carried out in 2001 and 2002 in lemon orchards in south-western New South Wales to assess the suitability of imidacloprid as a replacement for endosulfan in controlling the spined citrus bug (SCB), Biprorulus bibax Breddin (Hemiptera: Pentatomidae). The results showed that imidacloprid was at least as effective as endosulfan in controlling SCB, even when it was applied at a rate corresponding to half of its discriminate dose $(100 \%$ kill $)$. The application of imidacloprid did not reduce numbers of wasps and spiders foraging in tree canopies. However, imidacloprid was more toxic than endosulfan to parasitoids of the red scale and to lacewings. These negative effects can be minimised by timing the sprays in winter, when SCB adults congregate in neighbouring orange trees and the numbers of natural enemies of citrus pests are generally low.
\end{abstract}

The spined citrus bug (SCB), Biprorulus bibax Breddin (Hemiptera: Pentatomidae), is an important pest of lemons and mandarins in inland Australia (James, 1989). Both adults and nymphs feed on the fruit, resulting in internal staining, gumming, and fruit drop (Smith et al., 1997). A small number of bugs on a tree can cause a great deal of fruit loss. Hely et al.(1982) provided a summary of the biology and ecology of SCB. The eggs are laid on leaves, fruit or twigs in rafts of 4-36. In southern Australia, egg-laying starts in October and continues until late March. After hatching, the insect passes through five nymph stages before entering the adult stage. The life cycle takes $4-6$ weeks in summer but may take as long as 10-12 weeks in cooler months in the southern states. The adults over-winter in aggregations on nearby non-lemon citrus trees or on their native host the desert lime Eremocitrus glauca. A maleproduced pheromone is responsible for the aggregation (Oliver et al., 1992).

Currently, control of SCB relies heavily on the judicious use of endosulfan, an organochlorine compound. When used at a very low rate $(10-15 \mathrm{~mL} / 100 \mathrm{~L})$, endosulfan is very effective against SCB but is harmless to most other invertebrates found in citrus groves, including the natural enemies of SCB and other citrus pests (James, 1994). Unfortunately, it is inevitable that endosulfan will eventually be removed from use in citrus, as is the case in most other countries. In Australia, registration of endosulfan has been withdrawn from

Received for publication 6 May 2002. Accepted for publication 17 Oct. 2002. We wish to thank P. Morris, J. Morris, F. Ciccia, F. Vilaata, and J. DeQuino for kindly provide us with the trial sites, and $\mathrm{C}$. Bower for commenting on the draft version. This study is funded by Horticulture Australia and levy from the Australian citrus industry.

${ }^{1}$ To whom reprint requests should be sent. Telephone, 61-2-6951 2537; fax, 61-2-6951 2719; E-mail, jianhua.mo@agric.nsw.gov.au stone fruits and stringent regulations have been recently introduced for its use in other crops. Other currently registered chemicals for SCB control in citrus (e.g., malathion, supracide) are broad-spectrum, which destroy virtually all invertebrates in citrus orchards and create conditions for dramatic pest resurgences (including SCB). Prior to development of SCB IPM, up to six applications of malathion/ supracide per season were often needed to control SCB. Serious outbreaks of scale insects, mealybugs and aphids also resulted from these treatments.

Viable alternatives to endosulfan are clearly needed in anticipation of its withdrawal from Australian horticulture. From 1997 to 2001, a research project was carried out in southwestern New South Wales investigating some chemical and biological alternatives to manage SCB. As part of this project, three new generation insecticides known to be effective against hemiptera were evaluated: abamectin, fipronil, and imidacloprid. All three were found (James and Nicholas, 2000), but only imidalcoprid achieved satisfactory control of SCB in a small-scale field trial (Nicholas, 2000). This paper reports findings from subsequent large-scale field trials comparing the relative field performance of imidacloprid and endosulfan in controlling SCB and their relative impact on some important natural enemies of major citrus pests.

\section{Materials and Methods}

Trial description. Four large-scale field trials were conducted from Oct. 2001 to Mar. 2002 in lemon [Citrus limomum Risso. (Eureka)] orchards in the Riverina region of New South Wales. The test trees were 3.5-6 $\mathrm{m}$ tall and spaced at 5-7 $\mathrm{m}$ between rows and $3-5 \mathrm{~m}$ within rows. Two rates of imidacloprid (200 $\mathrm{g} \cdot \mathrm{L}^{-1}$ a.i.), $10 \mathrm{~mL} / 100 \mathrm{~L}$ (full-rate) and to be highly toxic to SCB adults and nymphs
$5 \mathrm{~mL} / 100 \mathrm{~L}$ (half-rate), were compared with endosulfan $\left(350 \mathrm{~g} \cdot \mathrm{L}^{-1}\right.$ a.i. $)$ at the industry standard rate of $57 \mathrm{~mL} / 100 \mathrm{~L}$. The higher of the two rates for imidacloprid corresponds to the discriminate dose (100\% kill) of the insecticide against SCB (James and Nicholas, 2000). A water-only treatment was provided as control in all but one trial. Applications of the treatments were made with air-blast sprayers at a spray volume of $10 \mathrm{~L} /$ tree.

Trial-1 was conducted from 25 Oct. to 24 Nov. 2000 in Leeton. Lemon trees from three neighbouring citrus farms (separated by $1-2$ $\mathrm{km}$ ) were used in this trial. The test trees were in six separate blocks (>100 m apart) containing from 60 to 453 trees. Fifteen plots of $60-100$ trees each were set up in the six lemon blocks. Where more than one plot was set up in a single block, the boundaries were chosen in such a way that each plot contained a similar number of trees and the adjacent plots were at least $8 \mathrm{~m}$ apart. Three treatments were tested in this trial: full-rate imidacloprid, standard-rate endosulfan, and water control. The trial was designed as unbalanced incomplete random blocks with each farm treated as a separate statistical block. There were 5 replicates per treatment.

The other three trials (Trial-2, Trial-3 and Trial-4) were not replicated. They were conducted simultaneously between $12 \mathrm{Feb}$. to 13 Mar. 2001 in three separate citrus farms in Griffith. The sizes of the plots were larger than in Trial-1, averaging 0.21 ha $(0.17-0.22$ ha). Trial-2 was conducted in two adjacent lemon blocks (94 and 245 trees). The larger block was divided into two equally sized plots with a one-row ( $5 \mathrm{~m}$ ) buffer. The smaller block was assigned as a plot by itself. Three treatments, full-rate imidacloprid, standard-rate endosulfan, and water were randomly assigned to the three plots. Trial-3 was conducted in a single block of 237 lemon trees. A single row of orange trees (Washington) separated the block into two equal size sub-blocks (114 and 123 trees). The two sub-blocks were assigned as two separate plots, with one plot treated with half-rate imidacloprid and the other with standard-rate endosulfan. Trial-4 was a single block of 418 lemon trees divided into three equal size plots with a one-row $(5 \mathrm{~m})$ buffer. Three treatments, full-rate imidacloprid, standard-rate endosulfan, and water were randomly assigned to the three plots.

Data collection. One week before the applications, 10 rectangular quadrats $(7 \times 3$ m) were set up on the orchard floor of each plot and raked clear of grass and debris. The quadrats were positioned around the bases of trees with the longer side parallel to the row. From the first day following treatment applications, the number of dead SCB adults and nymphs on the quadrats were counted daily for three consecutive days. Other dead invertebrates found each day on the quadrats were collected, identified to families and counted in the laboratory. Changes in the numbers of live SCB adults and nymphs during the trials were monitored by 0.5 -h searches of the canopies of the trial trees of each plot at 2-d before and $7,14,21$, and 28 -d after the spray. 
The impact of treatments on the parasitoids of the red scale (Aonidiella aurantii (Maskell)), parasitoids of the SCB, and on the abundance of parasitoids and predators in tree canopies was assessed in the three nonreplicated trials. For the impact assessment on red scale parasitoids, 30 red scale infested fruits were collected randomly from each plot $1 \mathrm{~d}$ before and 7, 14, 21, and $28 \mathrm{~d}$ after the spray. These fruits were taken to the laboratory and left in sealed containers in a constant temperature room $\left(25^{\circ} \mathrm{C}\right)$ for 4 weeks before being examined. The number of each parasitoid species emerged from each sample was recorded. For percentage parasitism of the red scales, a subsample of 100 mature female red scales was randomly drawn from each sample. These scales were examined under a stereo microscope for evidence of parasitism (emergence holes of the parasitoids or various stages of the parasitoids inside the scales). For impact assessment of the treatment on egg parasitoids of SCB, any egg batches found during the 0.5 -h search of live SCB in each plot were taken back to the laboratory and checked for parasitism. To supplement the field data, fresh egg batches from an SCB culture were placed in the trial plots and collected one week later. The impact of the treatments on the abundance of parasitoids and predators in tree canopies was assessed with sweep netting (net opening diameter $40 \mathrm{~cm}$, length $55 \mathrm{~cm}$ ). One hundred random sweeps were made in the tree canopies of each plot at $1 \mathrm{~d}$ before and 7,14, 21, and $28 \mathrm{~d}$ after the spray. The contents of the sweeps were emptied into sealable plastic bags and identified to families in the laboratory.

Data analyses. Analysis of variance (ANOVA) of the data from the unbalanced incomplete block design trial were performed with S-PLUS (Everitt, 1994), and the means separated by Duncan's multiple range test (Steel and Torrie, 1960).

\section{Results}

Knockdown effects. Pretreatment levels of SCB abundance in the three treatments in Trial-1 were similar $(\mathrm{F}=0.206$, $\mathrm{df}=2,10$, $P=0.22$ ) (Fig. 1). After the spray, a total of 1168 SCB adults and nymphs were found dead on the orchard floor, of which over $99 \%$ were from either imidacloprid-treated or endosulfan-treated plots (Fig. 1). The differences among the three treatments were significant $(\mathrm{F}=5.09, \mathrm{df}=2,10, P=0.03)$. However, there was no significant difference between the number of dead SCB found in imidacloprid-treated plots and those in endosulfan-treated plots $(P$ $>0.05$, Duncan's multiple range test).

The knockdown effects of the two chemicals in the three nonreplicated trials (Trial-2, Trial-3, and Trial-4) are shown in Fig. 1. All three trials indicated similar levels of efficacy of imidacloprid against SCB as compared with endosulfan, even when imidacloprid was applied at the half-rate (Trial-3).

Apart from SCB, 87 other dead arthropods were found on the ground quadrats after the spray in Trial-1, including 14 green lacewings
(Neuroptera: Chrysopidae), four ladybeetles (Coleoptera: Coccinellidae), three wasps (Hymenoptera: Ichneumonidae) and two spiders (Arandeida: Salticidae). Most of the dead lacewings (79\%) were found in plots sprayed with imidacloprid. No dead beneficial insects or spiders were collected from the ground quadrats in the three nonreplicated trials.

Effects on local SCB populations. Adult numbers in three of the four trials, Trial-1, Trial-2 and Trial-3, crashed from a minimum of 10 to a maximum 1 per plot per 0.5 -h search within the first week of the sprays in both endosulfan and imidacloprid-treated plots (Fig. 2). The populations largely remained at the low levels for the entire 4-week postspray period. There were slight increases of adult numbers in the two treatments in Trial- 1 at the end of the trial period. However, the numbers were still $70 \%$ to $75 \%$ below their respective pretreatment levels. Reductions of the adult numbers following the spray were also observed in the controls although at much lower rates. The effect of imidacloprid on the live adult population in Trial- 4 cannot be assessed as no adults were found in the treatment before the spray. Pretreatment levels of the adults in the other two treatments in this trial were too low ( $\leq 3$ per 0.5 -h search) to allow meaningful analysis.

The application of imidacloprid and endosulfan effectively wiped out SCB nymphs in Trial-3 and kept the nymph numbers at nondetectable level for the rest of the trial period (Fig. 3). Similar effects of the two chemicals were shown in Trial-2 and Trial4. However, the results were not as conclusive due to relatively low pretreatment levels of the nymphs in one or both of the chemical treatment ( $\leq 3$ per 0.5 -h search). In the replicated trial (Trial-1) live SCB nymphs were detected only in the imidacloprid treatment before the

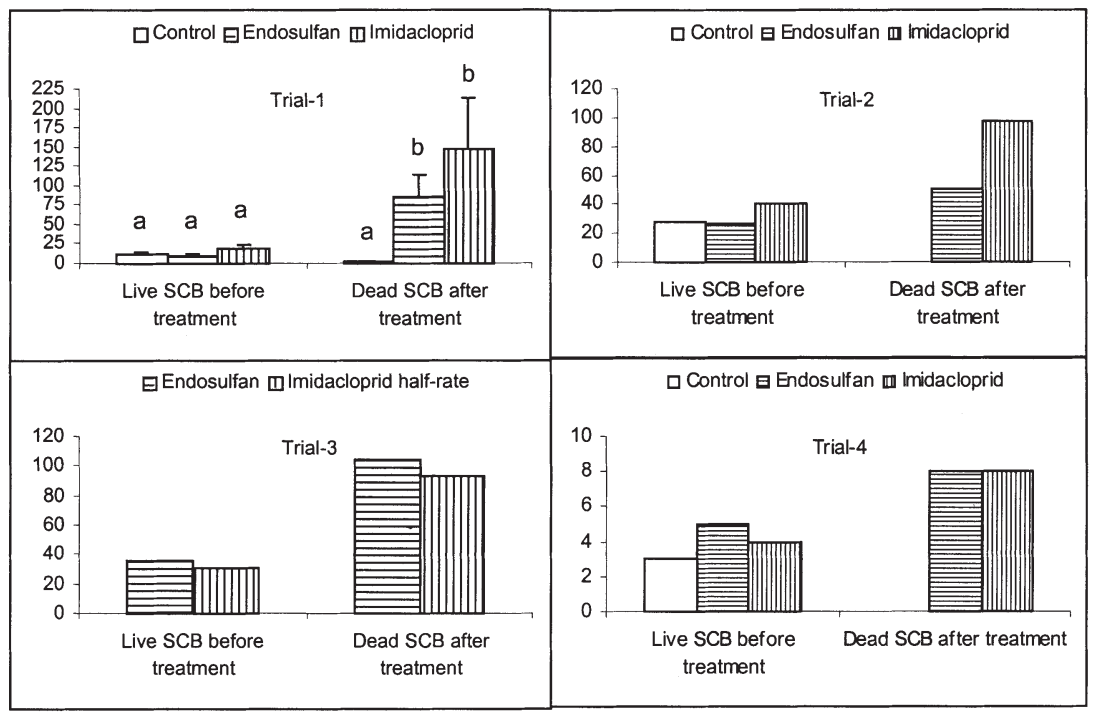

Fig. 1. Average number of live SCB adults and nymphs found per plot during random searches of trees 1 week before the spray and numbers of dead SCB adults and nymphs found on the orchard floor $3 \mathrm{~d}$ after the spray. Trial-1 was a replicated trial, whereas the other three trials were nonreplicated. Error bars show the standard errors of the means. Bars bearing the same letter were not significantly different within groups by Duncan's multiple range test at $P=0.05$.

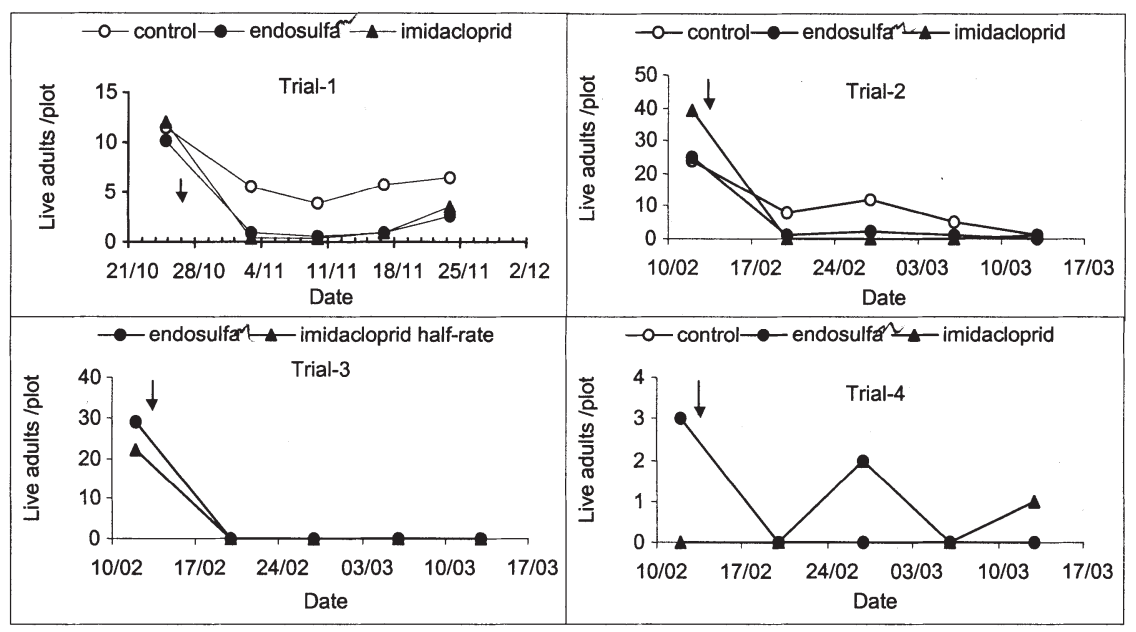

Fig. 2. Average number of live SCB adults found per plot during random searches of trees before and after the sprays. Arrows indicate the spray date. 
spray. After the spray the number of nymphs in the treatment decreased by $>50 \%$. In the 4-week postspray period, nymph numbers were consistently higher in the control than in the two chemical treatments. At the end of the trial period, nymph numbers in all three treatments increased to above the pretreatment levels.

Effects on beneficial insects. Parasitism of the red scale in the three nonreplicated trials ranged from $1 \%$ to $44 \%$. Most of the parasitism was by Comperiella bifasciata Howard (65\%) and Aphytis sp. (29\%). All three trials showed initial reductions of parasitism following the spray in both imidacloprid and endosulfantreated plots (Fig. 4). In Trial-3 and Trial-4 the parasitism level in imidacloprid-treated plots did not recover to the pretreatment levels, whereas that in endosulfan-treated plots rose to above the pretreatment levels within 3 weeks of sprays in all three trials. In Trial-2, the parasitism level was much lower in the imidacloprid treatment than in the endosulfan treatment for most of the postspray period. Reduction of red scale parasitism after the spray was not evident in the control.

The number of red scale parasitoids emerged from the fruit samples decreased sharply in imidacloprid-treated plot after the spray in all the three trials and stayed low for 3 weeks in two trials (Fig. 4). By contrast, the reduction in the emergence of red scale parasitoids by endosulfan was evident only in Trial-3. Even in this trial the emergence level partly recovered 2 weeks after the spray. In the other two trials the number of emerged parasitoids remained steady (Trial-4) or increased (Trial-2) after the application of endosulfan. The emergence of red scale parasitoids in the control increased after the spray in Trial-2 and initially decreased and then increased after the spray in Trial-4. Both imidacloprid and endosulfan appeared to suppress the emergence of male red scales within the first week of spray (Fig. 5).

A total of 13 field egg batches of SCB were collected in the three trials. Four egg batches were collected before the spray, all parasitised by Anastatus biproruli. Of the nine egg batches collected after the spray, only one was parasitised. This egg batch was collected from the control site 4 weeks after the spray. Twenty egg batches from the laboratory culture were put out in imidacloprid and endosulfan-treated plots. Two of the $20 \mathrm{egg}$ batches, one put out 3 weeks after the spray and the other 4 weeks after the spray, were parasitised by A. biproruli. Both were from the imidacloprid treatment.

Beneficial insects collected from sweep net sampling in the canopies of trial trees consisted of mainly wasps (Hymenoptera: Braconidae, Ichneumonidae) (74\%) and spiders (Araneida: Salticidae, Theridiidae, Thomisidae, Clubionidae) (20\%). A few ladybeetles (Coleoptera: Coccinellidae) and green lacewings (Neuroptera: Chrysopidae) were also collected. Neither imidacloprid nor endosulfan showed apparent adverse effects on the abundance of foraging wasps (Fig. 6). Although the number of spiders in two of the

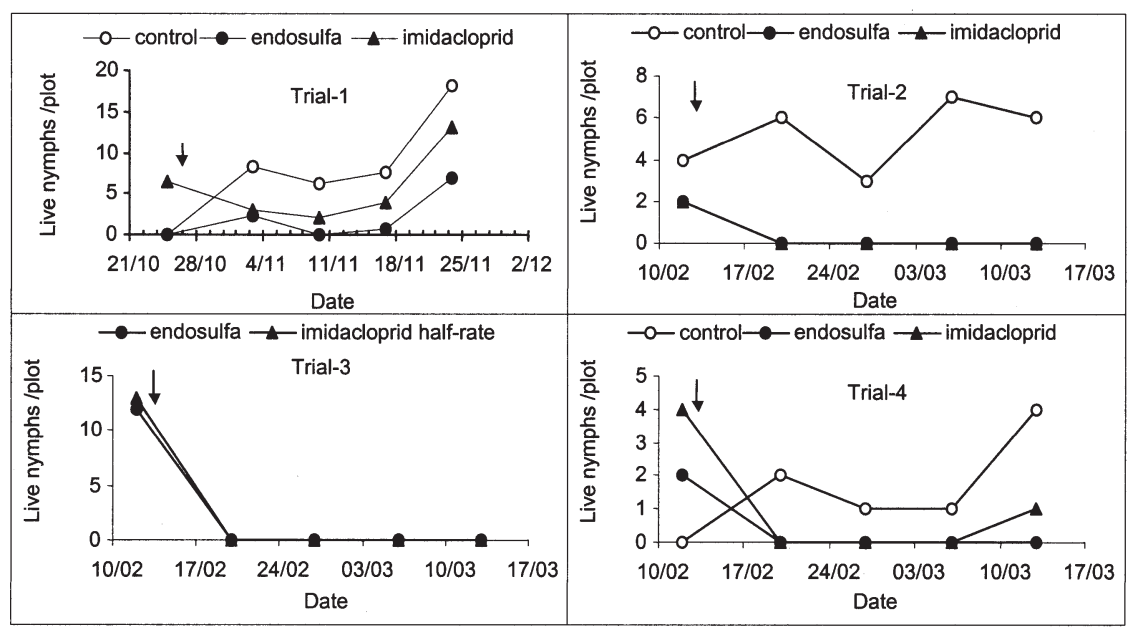

Fig. 3. Average number of live SCB nymphs found per plot during random searches of trees before and after the sprays. Arrows indicate the spray date.

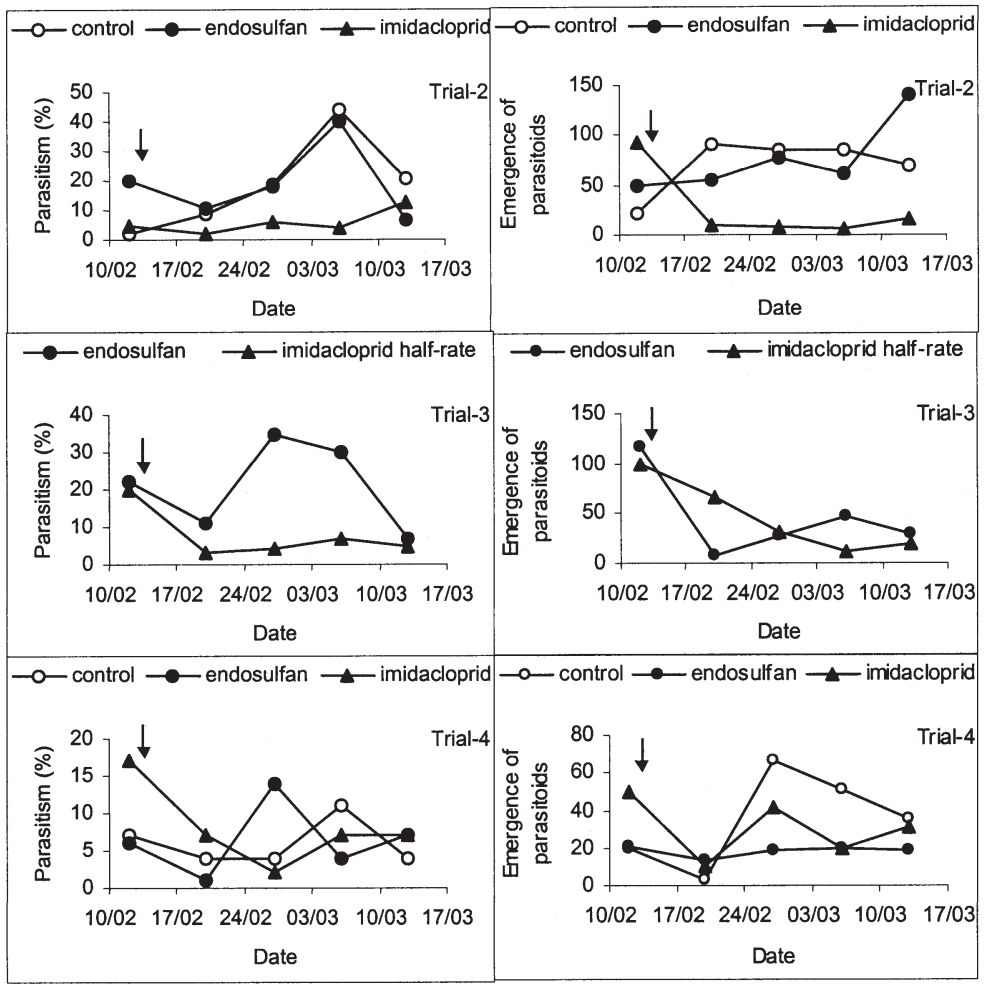

Fig. 4. Parasitism level of red scales and number of red scale parasitoids emerged per sample (30 red scale infested fruits) before and after the sprays. Arrows indicate the spray date.

three trial sites decreased 1 week after the spray in the imidacloprid-treated plot, similar decreases were also observed in the control plot (Fig. 6).

\section{Discussion}

Imidacloprid is a chemical from the chloronicotinyl group of insecticides. It has a novel mode of action, has low toxicity to warmblooded animals and excellent systemic and residual properties (Seidel, 1999). Imidacloprid is particularly effective against sucking insects such as aphids, leafhoppers, thrips and whiteflies (Altmann and Elbert, 1992; Seidel and Matthiessen, 1999). Because of its novel mode of action, it is effective against pest populations that are resistant to organophosphates, carbamates and pyrethroids.

Results from the four field trials demonstrated that imidacloprid was at least as effective as endosulfan in controlling SCB, even when it was applied at a rate corresponding to half of its discriminating dose. When no insecticide-free plot was provided, one application of imidacloprid was sufficient to reduce 


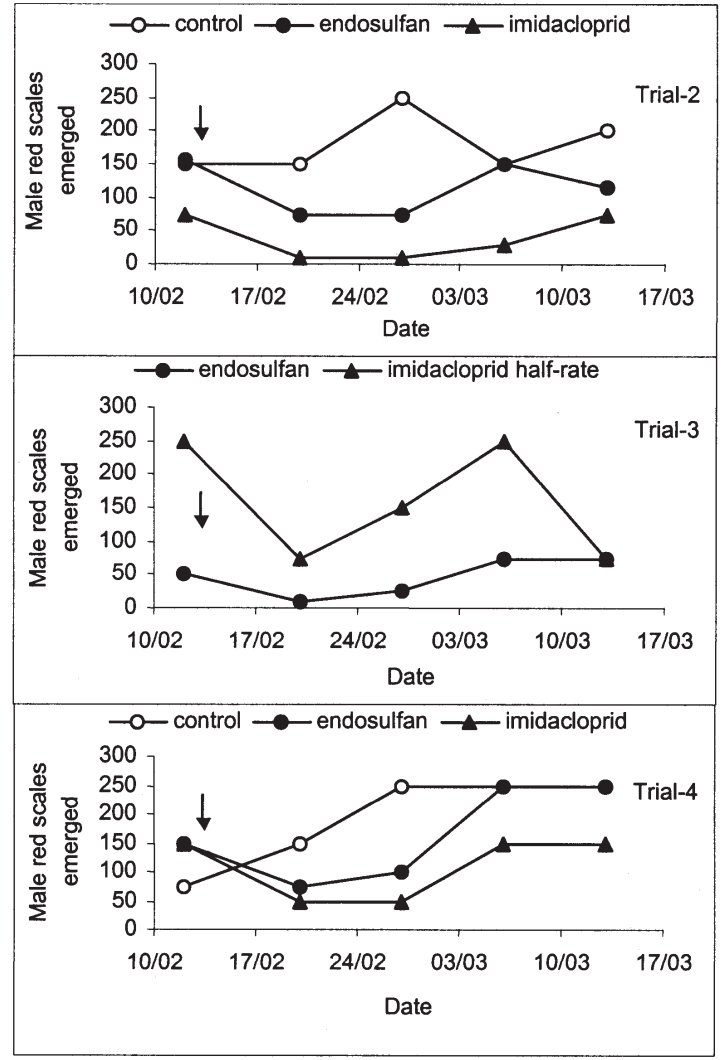

Fig. 5. Numbers of adult male red scales emerged per sample (30 red scale infested fruits) before and after the sprays. Arrows indicate the spray date.

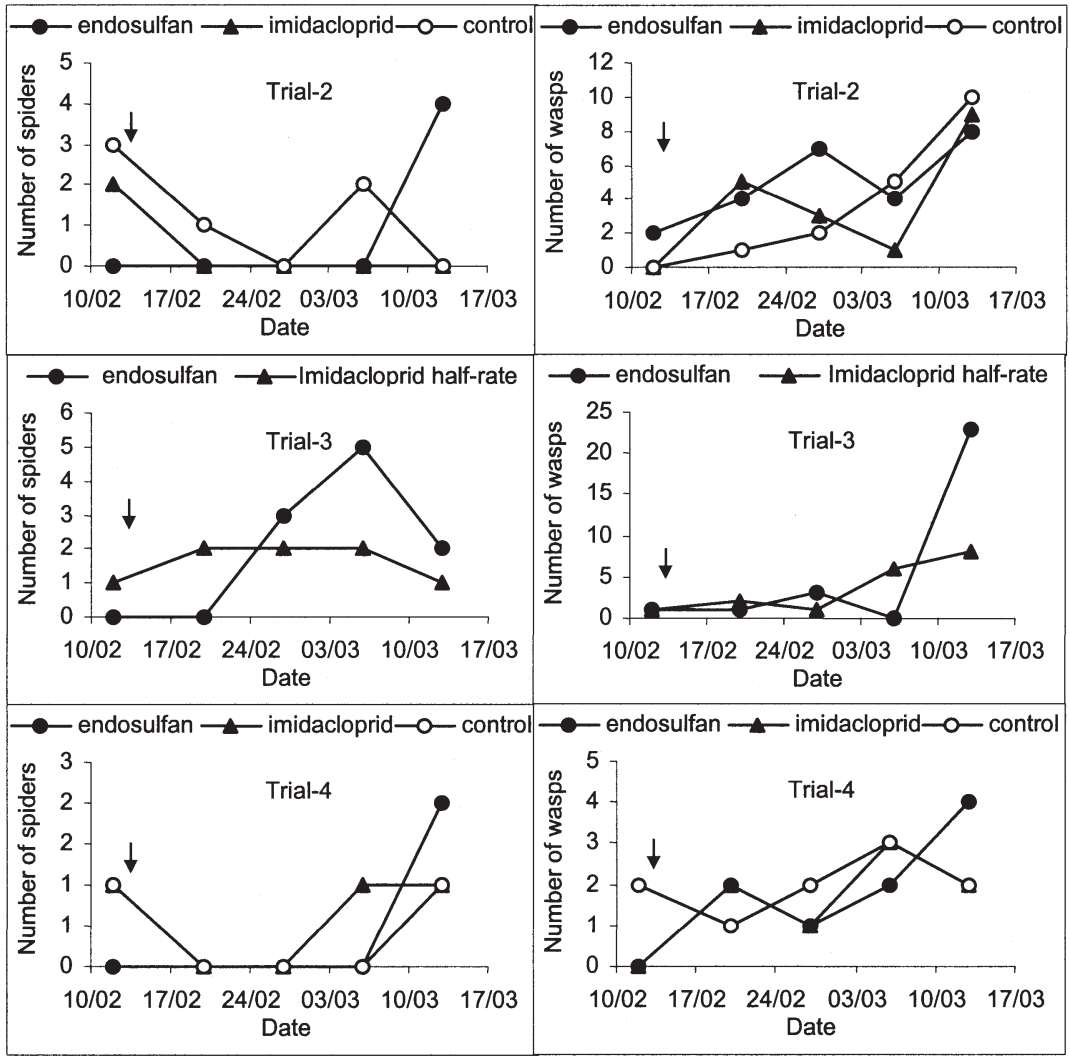

Fig. 6. Numbers of spiders and wasps caught from tree canopies by sweep netting in the three nonreplicated trials. Arrows indicate the spray date. the local SCB populations to an undetectable level for 4 weeks. The actual nondetection period could be longer as the populations were not monitored beyond 4weeks after the spray. However, when areas of the orchard were left unsprayed for control plots, the numbers of SCB adults and nymphs started increasing much sooner due to frequent inter-plot movements of adults (personal observation). The same reason explains why the numbers of live adults in the control plots decreased following the spray. Since the total number of SCB adults in an orchard was greatly reduced by the sprays, fewer adults were available to move into the control plots.

Many insect pests of citrus are normally kept at low levels by their natural enemies (Smith et al., 1997). A good example is red scale, one of the most important pests of citrus. This study showed that imidacloprid was harder on red scale parasitoids than endosulfan in terms of reducing parasitism rate and the emergence of parasitoids. However, this negative effect of imidacloprid may be partially offset by its positive effect in suppressing the emergence of male red scale. It was also observed that imidacloprid was more toxic to lacewings than endosulfan. The relative toxicity of imidacloprid and endosulfan to the egg parasitoids of SCB could not be determined due to the low number of egg batches collected from the trial sites. However, the results suggest that some SCB egg parasitoids survived the imidacloprid treatment, as $10 \%$ of the sentinel egg batches put out in the treatment after the spray were parasitised. This study did not detect any apparent adverse effects of imidacloprid on the abundance of foraging wasps and spiders foraging in tree canopies.

In view of the findings from this study, it is suggested that imidacloprid be considered as a short-term replacement for endosulfan if the latter is suddenly withdrawn. The possible negative effect of imidacloprid can be minimized by carefully timing the spray, using lower rates, and spraying only in areas of the orchard where SCB density is high. Schmidt and Hartmann (1999) noted that applying imidacloprid at the time of shoot formation had little effect on beneficial insects in apple orchards. For the control of SCB, the best time for spraying imidacloprid appears to be winter, when SCB adults congregate in orange trees surrounding lemon blocks (James, 1989). The advantage is that farmers can spot-treat selected trees instead of spraying the whole orchard and thus avoid large impact on local populations of beneficial insects. Impact on beneficial insects can also be reduced by using lower rates of imidacloprid (eg. $<5 \mathrm{~mL} / 100 \mathrm{~L}$ ). This study showed that halving the application rate of imidacloprid did not reduce its efficacy against SCB. It is possible that the rate can be reduced further without compromising the control efficiency, as has been demonstrated for the control of SCB with endosulfan (James, 1993, 1994). Long-term solutions to the withdrawal of endosulfan, however, will require more selective insecticides. 


\section{Literature Cited}

Altmann, R. and A. Elbert 1992. Beneficial-friendly use of Confidor $\mathrm{R}$ for integrated crop protection in fruit cultivation. Spec. issue: Imidacloprid. Pflanzenschutz Nachrichten Bayer 52:347-359.

Everitt, B.S. 1994. A handbook of statistical analyses using S-PLUS. Chapman \& Hall, London.

Hely, P.C., G. Pasfield, and J.G. Gellatley. 1982. Insect pests of fruit and vegetables in NSW. Inkata Press, Melbourne.

James, D.G. 1989. Population biology of Biprorulus bibax Breddin (Hemiptera: Pentatomidae) in a southern New South Wales citrus orchard. J. Austral. Entomol. Soc. 28:279-286.

James, D.G. 1993. Toxicity of endosulfan to spined citrus bug, Biprorulus bibax, and some of its egg parasitoids. Plant Protection Quart. 8:54-56.
James, D.G. 1994. The development of suppression tactics for Biprorulus bibax (Heteroptera: Pentatomidae) as part of an integrated pest management programme in citrus in inland south-eastern Australia. Bul. Entomol. Res. 84:31-38.

James, D.G. and A.H. Nicholas 2000. Bioassay results for alternative insecticides. Milestone Rpt. 2, Project CT97010 (Development of an alternative to endosulfan in the IPM of spined citrus bug). Hort. Australia.

Nicholas, A.H. 2000. Field evaluation of insecticides. Milestone Rpt. 3, Project CT97010 (Development of an alternative to endosulfan in the IPM of spined citrus bug). Hort. Australia.

Oliver, J.E., J.R. Aldrich, W.R. Lusby, R.M. Waters, and D.G. James. 1992. A male-produced pheromone of the spined citrus bug. Tetrahedron Lett. 33(7):891-894.
Schmidt, H.W. and J. Hartmann 1999. Beneficialfriendly use of Confidor for integrated crop protection in fruit cultivation. Special issue: Imidacloprid. Pflanzenschutz-NachrichtenBayer. 52(3):347-359.

Seidel, J.E. 1999. A new approach to the control of earth mites using Gaucho R seed dressing, $\mathrm{p}$. 132-136. In: J.N. Matthiessen (ed.). Proc. 7th Australasian Conf. Grassland Invertebrate Ecol. CSIRO Entomol. Wembley, Australia.

Smith, D., G.A.G. Beattie, and R. Broadley (eds.) 1997. Citrus pests and their natural enemies: Integrated pest management in Australia. Queensland Dept. of Primary Ind. and Hort. Res. and Devel. Corp., Brisbane.

Steel, R.G.D. and J.H. Torrie. 1960. Principles and procedures of statistics with special reference to the biological sciences. McGraw-Hill, London. 